\title{
Article
}

\section{La recherche en partenariat : entre fiction et friction}

\author{
Christophe Toussaint Soulard ${ }^{\mathrm{a}}$, Claude Compagnone ${ }^{\mathrm{b}}$, Bruno Lémery ${ }^{\mathrm{b}}$ \\ a Géographe, INRA, UMR951 Innovation, Campus INRA/Agro-M, 2 place Pierre Viala, 34060 Montpellier Cedex 1, France \\ b Sociologues, ENESAD-INRA, UR718 LISTO, 26 bd du Docteur Petitjean, BP 87999, 21079 Dijon Cedex, France
}

Les conditions de la production de connaissances dans un contexte d'action publique ont plusieurs fois été abordées dans NSS. Cet article apporte sur le sujet des éléments nouveaux, à partir d'une étude empirique où sont comparés trois exemples de recherche en partenariat. Ces dernières impliquent, pour démarrer, une certaine fiction : la croyance, partagée entre chercheurs et partenaires, qu'en collaborant, ils peuvent fabriquer des connaissances utiles. Chemin faisant, elles entraînent, entre protagonistes, des frictions profitables au déroulement du travail. Fiction et friction sont constitutives de la recherche en partenariat; c'est la thèse ici défendue.

La Rédaction

\section{Mots-clés :}

recherche en partenariat; médiation; interdisciplinarité ; conduite de projet

Résumé - L'article explore les enjeux et les pratiques de recherches interdisciplinaires en partenariat. À partir d'une lecture du déroulement de trois projets de recherche, portant sur l'agriculture face à des enjeux sanitaires ou environnementaux, les auteurs analysent l'effet sur la production scientifique des relations établies avec des acteurs du développement. L'hypothèse est que ce partenariat s'inscrit dans une double réalité : l'une, "fictionnelle», qui postule que chercheurs et partenaires s'allient tout en évitant de se confronter vraiment, ce, afin de maintenir le cours des choses ; l'autre, «frictionnelle », qui surgit lorsque, dans une phase d'investissement plus poussé des acteurs, des désaccords apparaissent, ce qui réoriente le cours des événements. Les résultats révèlent la mise en tension de cette double réalité dans chacun des projets. Leur comparaison fait ressortir trois points-clés de la conduite de ces recherches : le degré de constitution et la force du «projet " propre des acteurs impliqués, le rôle pivot d'individus médiateurs et l'attention qu'il convient de porter aux reconfigurations éventuelles du partenariat qu'impliquent les évolutions mêmes du projet.

\section{Keywords:}

partnership research; mediator; management of project; interdisciplinarity
Abstract - Partnership research: from fiction to friction Our paper deals with the issues and practices at stake in the interdisciplinary partnership research field. Taking as a starting point the observation of three on-going research projects confronting agriculture with environmental or sanitary issues, the authors analyse the effects produced, in terms of scientific results, by the relationships established among researchers and development partners. The hypothesis is that the partnership is based on a two-sided reality: fiction, on the one hand, goes with the assumption that while researchers and partners do consider each other as allies, they consistently avoid confrontation in order to let things run their course. Friction, on the other hand, may arise with disagreement and thus alter the course of events. As revealed by the results of the study, this two-sided reality is held in tension within each project. Their comparison brings out three key points required in the management of such projects: the degree of solidarity between the groups concerned, the kingpin role of mediators, and the manner in which the research is conducted to meet changes in the partnership.

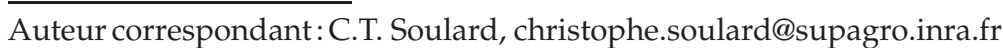




\section{Questions sur les recherches «en partenariat »}

Dans le domaine des recherches sur l'agriculture, l'environnement et le développement rural, les responsables des établissements de recherche et des organismes de développement prônent la nécessité et les vertus d'une recherche conduite «en partenariat » entre chercheurs et acteurs socioprofessionnels ${ }^{1}$ (Sebillotte, 1996; Béranger et al., 2002 ; Hervieu et al., 2003). Le partenariat apparaît dans ce discours comme le moyen qui doit permettre de développer des travaux socialement utiles et utilisables dans le cadre d'un dialogue effectif entre "science et société ». Cette conception postule que les acteurs partenaires de la recherche, discutant à égalité avec des chercheurs et directement associés à des choix de recherche, sont en mesure de tirer réellement parti de connaissances originales, à la fois scientifiquement fondées et répondant à des questions pratiques, dès lors qu'ils ont la possibilité d'intervenir dans leur production même. De leur côté, les chercheurs sont supposés pouvoir allier recherche fondamentale et intérêt pour un travail appliqué et impliqué, compétences disciplinaires pointues et aptitude au dialogue.

Si ce discours est bien générateur de recherches d'un genre particulier, il n'en reste pas moins que celles-ci ont une reconnaissance encore mal assurée au sein des institutions de recherche. Cet état de choses renvoie à la place relativement marginale qu'elles occupent dans une production scientifique globale dont les critères d'excellence tendent à autonomiser le fonctionnement de la science par rapport à celui de la société (Gadille et d'Iribarne, 2001). Il tient aussi au fait que les conditions de mise en œuvre de ces recherches restent, le plus souvent, trop peu réfléchies et trop peu explicitées (Albaladejo et Casabianca, 1997). Il existe, en effet, une certaine sousestimation, par leurs promoteurs et leurs auteurs, de l'importance, dans de tels travaux, des jeux d'acteurs induits par les différences de logiques portées par les parties impliquées. Cette sous-estimation se traduit par une attention insuffisante aux dispositifs - c'est-à-dire aux agencements de relations et d'opérations - à mettre en place pour parvenir aux résultats escomptés (Barbier, 1998). Une telle situation peut conduire à disqualifier ce type de travaux, soit parce que le partenariat devient simplement une sorte $d^{\prime}$ habillage de recherches qui continuent à fonctionner sur un mode classique, soit parce que le partenariat, dès lors qu'il acquiert une certaine réalité, entraîne une complexification de la situation qui peut freiner le processus de recherche, voire le détourner vers de simples actions de développement.

1 Cet article présente des travaux issus du programme de recherche Pour et sur le développement régional (PSDR) porté par l'Inra. Il s'agit d'une réflexion sur les pratiques de recherche en partenariat promues dans ce programme.

\section{Une hypothèse}

Face à ce constat, notre hypothèse, fondée sur l'expérience que nous avons pu acquérir de ce genre d'activité, est que des recherches en partenariat réellement consistantes supposent la mise en tension de deux moments, à la fois antinomiques et pourtant également nécessaires, que sont la «fiction » et la «friction ». Il y a fiction dans le sens où chercheurs et partenaires ne peuvent échapper à des situations qui correspondent à une confrontation faible : il n'y a pas de véritable débat qui s'instaure dans la mesure où les objets d'un tel débat ne sont pas « déjà là ", d'où un certain attentisme inévitable. Ils doivent alors contribuer à la coopération visée, moyennant un jeu d'interprétations et d'anticipations réciproques de ce qu'il faut faire pour que l'(inter)action se poursuive " quand même». Il y a friction dans le sens où une telle (inter)action entre des parties prenantes qui y sont engagées avec des logiques différentes - et qui doivent rester distinctes pour que la confrontation soit véritablement productive - ne peut faire l'économie de désaccords et de jeux stratégiques toujours susceptibles de contrarier le processus même de coopération visé.

Dans ces conditions, la productivité de recherches en partenariat nous semble dépendre très directement de la façon dont ces moments s'agencent, compte tenu de certains effets de dispositifs plus ou moins favorables à la concrétisation des objectifs que ces recherches se donnent. Pour avancer dans la compréhension de tels effets, il convient alors d'explorer les rapports effectifs des acteurs engagés dans des projets de recherche en partenariat, afin de dégager comment ces rapports peuvent jouer sur ce que produisent ces projets, en fonction de la manière dont ils s'organisent et sont organisés, de manière plus ou moins délibérée (Lémery et al., 1997). Dans cet article, c'est une telle approche que nous proposons, en nous appuyant sur une analyse transversale de travaux menés dans le cadre du programme PSDR Bourgogne (Encadré 1).

\section{Une lecture croisée de trois dispositifs de recherche en partenariat}

Ces travaux correspondent à trois projets dans lesquels nous avons été directement impliqués. Ils portent sur l'étude de transformations des pratiques d'agriculteurs, appréhendées dans des contextes de production différents. Nous considérons qu'il s'agit bien de recherches en partenariat dans la mesure où les trois projets reposent sur la mise en place d'un système d'engagements réciproques entre des chercheurs, insérés dans des collectifs de recherche divers, et des acteurs régionaux, conviés à participer au déroulement des recherches (Tab. 1).

Pour autant, la mise en place de ces partenariats ne s'appuie pas sur un modèle de recherche prédéterminé. 


\section{Encadré 1. Le «partenariat » dans le programme de recherche PSDR (deuxième programme 2001-2005)}

Le programme de recherche Pour et sur le développement régional (PSDR) a été initié par Michel Sebillotte, à l'Inra, pour soutenir des travaux de recherche d'un type nouveau. L'objectif est d'inciter chercheurs et acteurs régionaux à s'engager, en partenariat, dans des recherches co-construites, c'est-à-dire identifiées à partir de questions de développement jugées prioritaires pour une région (questions de la pratique), puis transformées en questions de recherche. Le dispositif régional prévoit un partenariat à deux niveaux :

- un «partenariat de programme» constitué d'un consortium d'organismes de recherche et de développement (œuvrant dans les domaines de l'agriculture, du rural et des industries agroalimentaires), réunis grâce à une convention-cadre fixant les objectifs de la coopération sur la durée du programme. Parmi ces organismes, les financeurs principaux du programme PSDR 2 sont les conseils régionaux et l'Inra ;

- un «partenariat de projet» formé par l'ensemble des acteurs parties prenantes d'un projet de recherche sur une thématique précise. À ce niveau, qui renvoie aux situations étudiées dans cet article, le partenariat entre chercheurs et acteurs régionaux ne fait pas l'objet d'une formalisation selon des objectifs déterminés au départ. Il se construit avec la dynamique du projet, suivant des rythmes et des intensités très variables selon les projets.

L'évaluation ex post de ce programme (Bailly et al., 2005) a révélé les limites d'un partenariat de projet peu formalisé, dans la mesure où un simple affichage pouvait permettre de justifier des recherches peu spécifiques. Mais le caractère procédural de la démarche partenariale a permis aussi de voir des avancées quand chercheurs et acteurs régionaux ont réussi à établir, chemin faisant, de nouveaux régimes de coopération (d'Iribarne, 2005).

Tableau 1. Les acteurs des trois projets de recherche étudiés.

\begin{tabular}{|c|c|c|c|}
\hline \multirow{2}{*}{ Projets } & Flexibilité & Biobacs & Viticulture \\
\hline & $\begin{array}{l}\text { La flexibilité des exploi- } \\
\text { tations d'élevage charolais } \\
\text { face à la crise de la filière bo- } \\
\text { vine (Lémery et al., 2003) }\end{array}$ & $\begin{array}{l}\text { L'efficacité d'installations de } \\
\text { traitement des effluents phy- } \\
\text { tosanitaires : les biobacs } \\
\text { (Fournier et al., 2004) }\end{array}$ & $\begin{array}{l}\text { Les dynamiques de change- } \\
\text { ment des pratiques viticoles } \\
\text { (Compagnone, 2003) }\end{array}$ \\
\hline $\begin{array}{l}\text { Équipe de } \\
\text { recherche } \\
\text { du projet }\end{array}$ & $\begin{array}{l}\text { Collectif de recherche com- } \\
\text { posé de cinq scientifiques, } \\
\text { d'une doctorante et d'un } \\
\text { technicien }\end{array}$ & $\begin{array}{l}\text { Noyau de deux ingénieurs et } \\
\text { d'une doctorante faisant ap- } \\
\text { pel à des compétences scien- } \\
\text { tifiques externes }\end{array}$ & $\begin{array}{l}\text { Un enseignant-chercheur et } \\
\text { de nombreux stages d'étu- } \\
\text { diants ingénieurs et masters }\end{array}$ \\
\hline $\begin{array}{l}\text { Acteurs } \\
\text { régionaux } \\
\text { partenaires } \\
\text { du projet }\end{array}$ & $\begin{array}{l}\text { - Chambre régionale d'agri- } \\
\text { culture } \\
\text { - Responsables profession- } \\
\text { nels et techniciens des or- } \\
\text { ganismes d'élevage d'un dé- } \\
\text { partement } \\
\text { - Éleveurs en suivi d'exploi- } \\
\text { tation }\end{array}$ & $\begin{array}{l}\text { - Firme phytosanitaire } \\
\text { - Groupe régional d'action } \\
\text { contre les pollutions phyto- } \\
\text { sanitaires dans l'environne- } \\
\text { ment (le GRAPPE), animé } \\
\text { par la DIREN } \\
\text { - Agriculteurs expérimenta- } \\
\text { teurs }\end{array}$ & $\begin{array}{l}\text { - Coordination régionale des } \\
\text { recherches et expérimenta- } \\
\text { tions en chardonnay et pinot } \\
\text { (CRECEP) } \\
\text { - Agents de l'encadrement } \\
\text { technique viti-vinicole } \\
\text { - Viticulteurs enquêtés }\end{array}$ \\
\hline
\end{tabular}

Il ne s'agit pas, par exemple, de projets choisis pour leur caractère exemplaire de mise en œuvre d'une rechercheintervention, au sens d'Hatchuel (2000). Si la recherche dans ces trois projets a bien pour finalité l'action, elle se trouve insérée dans des dispositifs qui agencent des méthodes de recherche classiques, reposant soit sur l'expérimentation au laboratoire (projet Biobacs), soit sur l'enquête de terrain (projets Flexibilité et Viticulture), et des moments de rencontre entre chercheurs et acteurs partenaires du projet (Fig. 1). Étudier et comparer ces agencements nous semble alors intéressant, non pas pour dégager ou discuter les principes théoriques et les règles méthodologiques des recherches en partenariat ${ }^{2}$, mais pour mieux cerner les effets sur le processus même de recherche des interactions entre chercheurs et acteurs régionaux engagés dans un même projet.

${ }^{2}$ Voir Sebillotte (2000).
Une telle orientation apparaît particulièrement appropriée pour rendre compte de partenariats dont le cours est relativement imprévisible. Ceux-ci correspondent, en effet, à ce qu'Aggeri (2002) et Segrestin (2003) nomment, par opposition aux partenariats stabilisés, des partenariats d'exploration. Dans le premier cas, les objets du partenariat vont de soi et les problèmes à résoudre ne portent que sur des questions du type partage des risques et des bénéfices, division du travail ou moyens d'éviter des comportements opportunistes. Dans le second, les régimes de coopération relèvent d'une construction simultanée et incertaine faisant intervenir des objets divers : objets de recherche, objets de gouvernement et, à l'interface, objets de partenariat. Étudier la manière dont ces différents objets se construisent et s'agencent au cours du temps du projet suppose alors de mobiliser une épistémologie de l'action en train de se faire (Hatchuel, 2000), du type de celle qui permet 


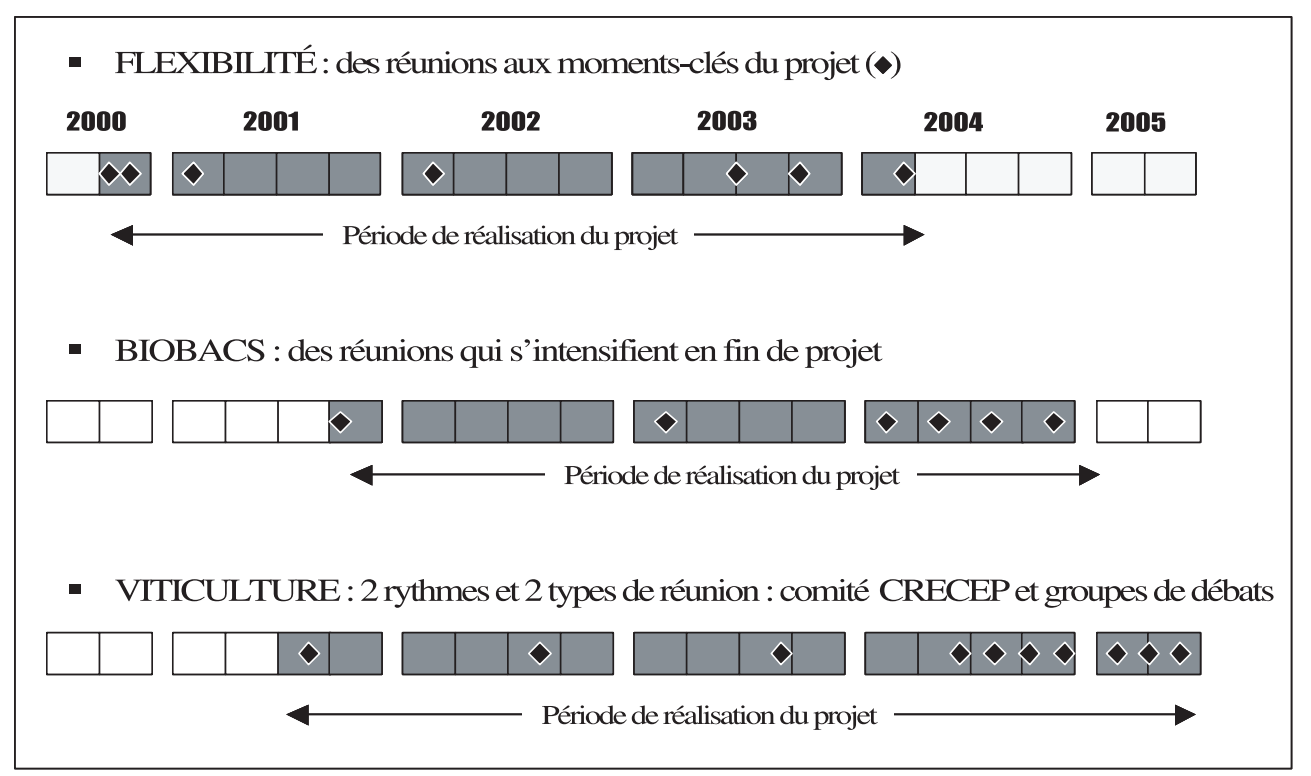

Fig. 1. Les rencontres chercheurs-partenaires régionaux dans le déroulement des projets.

d'étudier, par exemple, des processus d'apprentissage collectifs dans la conduite de situations de changement (Chia et Barbier, 1999; Chiffoleau et al., 2001).

\section{Un exercice de « retour » sur des pratiques de recherche}

Le matériau sur lequel repose notre analyse est constitué d'un corpus essentiellement formé des traces documentaires du déroulement des trois projets dont nous entendons rendre compte (lettres d'intention, exposés de leurs programmes, rapports intermédiaires et finaux, mais aussi courriers, notes et comptes rendus de réunions entre les équipes de recherche et les acteurs régionaux). Il est aussi marqué par la représentation que nous avons progressivement, et rétrospectivement, élaborée de ce qui s'était finalement passé dans des histoires dont nous étions les protagonistes.

Si cette sorte " $d$ 'introspection » est susceptible d'introduire des biais considérables dans l'interprétation de ces histoires, on insistera sur le fait que l'exposition même de l'exercice de réflexivité que nous avons entrepris de la sorte s'inscrit dans une perspective qui nous semble bien, cependant, relever d'un travail scientifique par la mise en débat de la pertinence et de la cohérence de l'interprétation proposée ici. On soulignera aussi que ce n'est pas pour rien que cet exercice a été mené collectivement, de façon à permettre l'expression entre les auteurs de divergences de vue et de conflits d'interprétation éventuels, forçant à expliciter ce que pouvaient signifier et avoir de commun trois expériences de recherche en partenariat qui, pour être subjectives, n'en expriment pas moins la réalité pratique de ce genre de recherche.
Pour rendre compte, alors, du produit de cette démarche, nous procéderons en deux temps. Nous présenterons, d'abord, l'histoire de chacun des trois projets ici considérés, en indiquant leurs objectifs initiaux ainsi que la nature et les conditions de mise en place du partenariat mobilisé pour leur engagement et en décrivant les évolutions de ces objectifs et de ce partenariat. Nous en effectuerons, ensuite, une analyse comparative.

\section{Trois projets de recherche en partenariat, trois histoires différentes...}

\section{Le projet Flexibilité}

Le projet Flexibilité portait sur l'analyse du rapport au changement des éleveurs charolais dans un contexte de crise de cette filière $\left(\mathrm{ESB}^{3}\right.$, baisse de la consommation de viande bovine, tensions entre logique de production de masse et logique de filières de qualité...), en vue $\mathrm{d}$ 'en tirer des enseignements pour le renouvellement $\mathrm{du}$ conseil aux éleveurs. L'équipe de recherche, constituée à cette fin, réunit des chercheurs de deux laboratoires de $\mathrm{I}^{\prime}$ Inra-SAD ${ }^{4}$. Affichant une ambition explicite de pluridisciplinarité, elle rassemble des zootechniciens, des économistes et des sociologues. À l'origine, le partenariat avec les professionnels de l'élevage se trouve mobilisé par les chercheurs de façon très volontariste. Souhaitant travailler sur «ce que changer veut dire » pour les éleveurs, ce qui suppose d'accéder à ce qui se passe dans

\footnotetext{
${ }^{3}$ Encéphalite spongiforme bovine.

${ }^{4}$ Institut national de la recherche agronomique, département Sciences pour l'action et le développement.
} 
les exploitations et dans le monde de l'élevage, ils sollicitent un soutien actif de ces professionnels. Ceux-ci, de leur côté, voient dans la demande qui leur est ainsi adressée une opportunité pour attirer l'attention de l'Inra sur les difficultés que connaît alors la filière. Au départ, il n'existe donc pas de problème vraiment "partagé ». On a plutôt affaire à une relative convergence d'intérêts.

Le dispositif de recherche adopté repose sur le suivi de quinze exploitations d'élevage, moyennant cinq enquêtes successives (une enquête exploratoire et quatre enquêtes thématiques correspondant aux différentes approches disciplinaires retenues). Au cours des six premiers mois, trois réunions sont organisées par les chercheurs pour définir et engager le travail avec les partenaires professionnels. C'est la recherche qui fixe le cadre des deux premières, au cours desquelles les discussions portent à la fois sur l'explicitation des intentions des chercheurs et sur les modalités de coopération souhaitées. Sans infléchir les visées initiales du projet, ces discussions portent surtout sur la contribution attendue des représentants des organismes professionnels, qui s'engagent alors à fournir des listes d'éleveurs choisis en fonction de critères proposés par la recherche. Une troisième réunion est organisée pour arrêter l'échantillon à enquêter. Elle se trouve marquée par une controverse entre professionnels sur la pertinence de certains «profils » d'éleveurs proposés (controverse dans laquelle les chercheurs n'interviennent que pour rappeler leur souci de couvrir la plus grande diversité possible de cas de figure).

Passée cette phase de démarrage, le rythme des rencontres avec les partenaires se réduit, le déroulement du projet étant essentiellement marqué par des échanges internes au collectif de recherche. La quatrième réunion ne se tient qu'un an plus tard, avec pour objectif de présenter les premiers résultats des enquêtes et de discuter des conséquences des connaissances produites en termes de conseil aux éleveurs. Dans les faits, ce dernier point n'est pas vraiment abordé. La séance est exclusivement centrée sur une restitution des premiers résultats de recherche, les partenaires se plaçant sur un registre d'évaluation de la pertinence à leurs yeux des comportements types d'éleveurs ressortant de l'analyse par les chercheurs. Il s'ensuit une longue période de dix-huit mois sans rencontre formelle avec les partenaires. Ce délai tient à des problèmes de disponibilité des chercheurs, mais aussi à des discussions internes à l'équipe de recherche sur la manière "d'intégrer » les différentes approches mobilisées, compte tenu de certaines divergences relatives à la signification de la notion de flexibilité et aux objectifs mêmes du projet. La situation devenant de plus en plus intenable - non pas que les partenaires aient manifesté une quelconque impatience, mais plutôt du fait de la montée d'une certaine «mauvaise conscience » du côté de la recherche, qui s'inquiète des effets éventuels de son absence de manifestation - une cinquième réunion avec les partenaires est donc organisée, sans que ces discussions aient été tranchées.

Cette réunion donne lieu, cette fois, à une véritable confrontation, portant sur la pertinence de certaines hypothèses faites, dans l'analyse des enquêtes techniques et économiques, sur ce qui peut être considéré ou non comme facteur de flexibilité des exploitations. Cet échange sur le fond a un double effet. Il aboutit à une nouvelle rencontre spécifiquement consacrée à l'examen conjoint, entre les partenaires du projet et les membres de l'équipe en charge de ses volets technique et économique, de ce que peut être un facteur de flexibilité, à la suite de quoi les chercheurs concernés élaborent une proposition de communication à un congrès scientifique. Il contribue également (via cette proposition) à relancer et à clarifier le débat interne à l'équipe de recherche sur les finalités mêmes du projet, ce qui permet d'amorcer véritablement le travail de synthèse des différents matériaux d'analyse accumulés. À l'issue de ce travail, une séance de restitution finale est alors organisée six mois plus tard, pour les éleveurs enquêtés, d'une part, et pour les techniciens et les responsables professionnels, d'autre part. Si la première réunion de restitution connaît un certain succès, ce n'est pas le cas de la seconde. La question à l'ordre $\mathrm{du}$ jour, toujours laissée en suspens, de l'accompagnement des éleveurs ne peut être abordée faute de participants. Dans ces conditions, et même si les chercheurs commencent à voir comment leurs résultats pourraient donner lieu à une traduction "opérationnelle », c'est à nouveau en fonction d'une logique interne au monde de la recherche que le projet s'oriente. Reportant à l'issue d'un événement à venir ${ }^{5}$ la concrétisation de ses ambitions initiales sur le conseil, l'équipe de recherche s'engage, moyennant un dernier séminaire interne, dans une activité de publication scientifique.

\section{Le projet Biobacs}

Le projet Biobacs porte sur l'étude d'un procédé de traitement des effluents phytosanitaires provenant des reliquats de pulvérisation (fonds de cuve dilués, eaux de lavage du matériel de pulvérisation) dont il faut éviter qu'ils soient rejetés dans le milieu naturel. Il est piloté par un microbiologiste de l'unité Inra-MGS ${ }^{6}$ et comporte deux volets de recherche : un volet expérimental consacré à des analyses microbiologiques et écotoxicologiques du fonctionnement des biobacs; un volet « enquêtes » portant sur l'étude des conditions de leur mise en place dans les exploitations agricoles.

\footnotetext{
5 Une journée «élevage » devant permettre de faire le point, avec les professionnels concernés, sur les acquis et les perspectives des différents projets menés dans ce domaine dans le cadre du programme PSDR Bourgogne.

6 Microbiologie et géochimie des sols.
} 
Ce projet s'engage dans la continuité d'une action préalable initiée par une firme phytosanitaire qui a introduit de Suède le procédé en France et qui a sollicité l'InraMGS pour analyser son efficacité à éliminer des résidus phytosanitaires. Le projet PSDR s'inscrit dans le prolongement d'un premier contrat entre l'Inra et cette firme. Il porte sur l'étude des risques environnementaux des biobacs et sur leur faisabilité dans les exploitations. Dans ce but, l'équipe de recherche intègre des compétences nouvelles en écotoxicologie, géochimie et sciences humaines. Le partenariat régional s'engage à la suite d'une journée «biobac info » au cours de laquelle le GRAPPE Bourgogne ${ }^{7}$, animé par la Direction régionale de l'environnement (DIREN), est identifié comme l'interlocuteur privilégié du projet PSDR.

Le partenariat qui s'établit de la sorte semble donc relever d'une alliance de circonstance. Pour le GRAPPE, le projet est un moyen d'accéder à des informations et des avis d'experts toujours utiles sur un sujet d'actualité brûlant (rédaction en cours d'un projet d'arrêté sur les effluents). Pour les chercheurs, ce partenariat est davantage tactique. La participation du GRAPPE insère le projet Biobacs dans une dynamique d'action régionale et permet d'affirmer le caractère public de cette recherche, jusqu'alors engagée sous l'impulsion d'une seule firme phytosanitaire.

Lorsque le projet débute, il est envisagé de réunir régulièrement les partenaires pour informer les uns et les autres de l'avancée du programme de recherche. Dans la réalité, les choses ne vont pas se passer comme cela. Sur les deux premières années du projet, une seule réunion entre l'équipe de recherche et les partenaires va avoir lieu. Celle-ci se tient peu après le lancement de l'opération. Des représentants du GRAPPE y participent. Cependant, la succession d'exposés des chercheurs présentant leurs intentions de recherche ne suscite pas de débats sur la conduite et les buts du projet. Par la suite, ce type de réunion ne se renouvelle pas. Une série d'imprévus va absorber le responsable du projet : certaines équipes ne démarrent pas leur travail malgré leurs engagements initiaux, et des réorganisations scientifiques au sein de l'unité MGS conduisent à distendre des liens au sein du collectif de recherche. Cette situation aboutit à un resserrement du projet Biobacs autour de son responsable et de la doctorante qu'il dirige sur ce thème.

Les contacts entre la recherche et les partenaires reprennent lors du lancement de l'étude de faisabilité des biobacs, deux ans après le lancement du projet. Une réunion avec des membres du GRAPPE est organisée pour se mettre d'accord sur les objectifs du travail d'enquête. Côté recherche, seuls le responsable MGS et le

\footnotetext{
${ }^{7}$ Groupe régional d'action contre les pollutions phytosanitaires dans l'environnement.
}

chercheur du LISTO $^{8}$ y participent. Ce dernier veille à inscrire l'étude de faisabilité dans une analyse plus globale des pratiques phytosanitaires dans les exploitations. Son souci est alors de faire que cette enquête, dont l'objet est très proche des préoccupations directes des partenaires, conserve bien une visée scientifique plus générique. La discussion avec les partenaires ne porte cependant pas sur ce point, mais davantage sur le choix du terrain d'étude. Elle débouche sur l'idée de lancer non pas un seul, mais deux mémoires d'étudiants : un sur la viticulture, où la question des effluents est vive; un autre sur les exploitations de grandes cultures et de polycultureélevage, mal connues de ce point de vue.

Trois réunions, rythmées par l'avancée des mémoires, vont ensuite se tenir. Progressivement, des liens plus forts s'établissent entre les deux chercheurs porteurs du projet Biobacs et les interlocuteurs du GRAPPE. Parmi ces derniers, les agents des structures publiques sont les plus actifs, très impliqués sur un dossier qui valorise la Bourgogne auprès de leur ministère de tutelle, en attente d'informations scientifiques qui étayent le projet d'arrêté. Les techniciens agricoles sont à la fois très présents aux réunions et discrets dans les discussions sur les biobacs, qu'ils jugent prématurées tant que «l'arrêté effluents » n'a pas paru. Dans ces conditions, quand, au terme de la troisième réunion consacrée à la restitution des deux mémoires, les chercheurs interpellent les partenaires sur ce qu'ils pensent faire des résultats, aucune réponse claire ne s'exprime immédiatement. L'appel de la recherche est cependant entendu par la DIREN, qui suggère de financer une opération de diffusion des résultats. Le LISTO réalise alors avec la DIREN et la chambre d'agriculture une journée régionale de restitution, à laquelle assistent 70 personnes, ainsi qu'une plaquette éditée à 1000 exemplaires (Danguin et al., 2004). Conçue dans une optique de restitution des résultats, cette opération produit un effet retour sur les chercheurs, qui doivent s'accorder sur un argumentaire scientifique cohérent, là où résultats expérimentaux et observations par enquêtes génèrent des débats, en interne, sur ce que la recherche peut délivrer comme «messages » à ses différents partenaires de l'action.

\section{Le projet Viticulture}

Ce troisième projet a été conçu pour appréhender les dynamiques de changement technique en viticulture bourguignonne, aussi bien au niveau des viticulteurs qu'au niveau des agents de l'encadrement technique. Il est porté par un sociologue, enseignant-chercheur associé au LISTO.

\footnotetext{
${ }^{8}$ Laboratoire de recherche sur les innovations sociotechniques et organisationnelles en agriculture.
} 
Le projet Viticulture est initié à partir d'une demande du représentant du développement agricole du comité de gestion de la CRECEP ${ }^{9}$, structure rassemblant l'interprofession viticole, les chambres d'agriculture et les composantes du pôle d'enseignement supérieur et de recherche dijonnais travaillant dans le domaine de la vigne et du vin. Animée et présidée par l'Inra, la CRECEP fournit au responsable du projet Viticulture des interlocuteurs privilégiés et organisés en tant que tels, via son comité de gestion, explicitement en charge du suivi de ce projet.

Cette situation est à l'origine d'un fonctionnement spécifique, du fait que le partenaire régional est ici le commanditaire du projet. Si le chercheur dispose bien d'une autonomie dans la conduite de ses travaux, les relations partenariales sont rythmées par le calendrier des réunions de la CRECEP. Le travail commence en 2001 par la production d'études préliminaires réalisées par des étudiants encadrés par le responsable du projet et restituées aux membres du comité de gestion. Il se poursuit en 2002 par un mémoire d'ingénieur portant sur la dynamique des changements de pratiques des viticulteurs d'une commune de Saône-et-Loire et sur le lien entre cette dynamique et la forme du réseau social constitué de l'ensemble des interactions existant entre ces viticulteurs. Les résultats de ce travail sont à nouveau restitués au comité de gestion. La discussion porte sur les similitudes et les différences entre les résultats de l'étude et les connaissances qu'ont les partenaires des viticulteurs d'autres communes viticoles. Mais aucun débat n'est vraiment engagé sur le genre de travail qui pourrait être opéré à partir de ce matériau pour l'établissement d'actions concrètes. L'année suivante, en 2003, la recherche se poursuit par un nouveau mémoire qui porte, cette fois, sur l'étude de l'encadrement technique des viticulteurs.

Attentive à un rythme de restitution des résultats, la CRECEP suscite la tenue d'un séminaire pour rendre compte de l'ensemble des résultats obtenus jusqu'alors et réfléchir aux modes d'action envisageables. Â ce séminaire, le responsable du projet présente les résultats des deux mémoires sur les viticulteurs et sur l'encadrement technique. Ce dernier travail révèle la diversité et la faible coordination des actions des agents apportant conseil auprès des viticulteurs. Surpris par ces données, le président de la CRECEP suscite une nouvelle réunion du comité de gestion pour réfléchir à des actions pouvant être rapidement mises en œuvre. Ce mouvement échappe au responsable du projet qui, bien qu'il le juge trop précoce, sent aussi que le rythme lent de la recherche ne joue pas en sa faveur et craint de perdre une part de sa légitimité si des éléments pour l'action ne sont pas produits sur un pas de temps convenable. À cette réunion, il est décidé de lancer une opération de concertation de

\footnotetext{
${ }^{9}$ Coordination des recherches et des expérimentations en chardonnay et pinot.
}

l'ensemble des agents de l'encadrement technique afin de réfléchir aux actions de conseil à développer.

La mise sur pied de cette opération de rechercheaction modifie le rythme d'activité de la recherche et l'implication des partenaires. Les rencontres avec eux passent alors à une réunion par mois, pour discuter collégialement des modalités pratiques de mise en œuvre du dispositif de concertation. Une chargée d'étude est recrutée pour six mois afin d'épauler le chercheur et $d^{\prime}$ assurer la liaison entre les uns et les autres lorsqu'un choix est à faire et une décision à prendre. Dans ces discussions, le chercheur incite les partenaires à s'appuyer sur les résultats antérieurement produits sur les modalités de changement des viticulteurs pour construire et ajuster l'opération de concertation visée. Cette démarche s'opère alors suivant un rythme qui découle des impératifs du développement et auquel la recherche doit se plier. À cette étape, c'est l'émergence d'éléments pour le développement qui est privilégiée dans la formalisation des données. Des comptes rendus sont envoyés régulièrement aux participants des groupes de concertation et aux partenaires du projet Viticulture. L'opération est clôturée par une restitution générale devant l'ensemble des participants durant l'été 2005. Ce n'est que dans l'année qui suit qu'une attention plus particulière est portée à la production scientifique; les liens avec le développement se relâchent.

\section{Analyse comparative}

Les trois chroniques illustrent les différences des trajectoires prises par les projets étudiés. Ces différences tiennent, bien évidemment, à certaines caractéristiques structurelles tant des collectifs de recherche concernés que des partenaires mobilisés. Côté recherche, la taille des équipes est très inégale, leur composition n'est pas la même, les statuts de leurs membres ne sont pas identiques, ce qui joue sur leur mode de fonctionnement. Côté partenaires régionaux, les modes d'engagement, les formes d'organisation, les degrés d'interconnaissance préalable et les positions qu'ils occupent (et peuvent prendre) dans le processus de travail sont également très variés. Mais, si ces éléments sont importants à considérer (et devraient donner lieu à un effort d'identification de leurs effets propres), il apparaît aussi que le cours des projets tient à leur histoire interne, à certains enchaînements d'événements qui conditionnent leur déroulement et leur orientation.

Nous allons donc essayer de montrer comment de tels enchaînements, et la manière dont ils ont pu être maîtrisés ou non, pour assurer l'avancée des choses, a contribué à la physionomie prise, dans nos projets, par les deux types de produits attendus d'une recherche en partenariat, à savoir des connaissances scientifiques validables et des connaissances « actionnables » (Argyris et Schön, 1978). 


\section{La production de connaissances scientifiques}

Les trois projets ici considérés sont tout juste terminés. Les premières publications scientifiques auxquelles ils ont donné lieu se limitent à des communications pour l'instant soumises seulement (à une exception près) à des revues scientifiques ${ }^{10}$. Il ne saurait alors être question de prétendre mettre en relation leur valeur intrinsèque (difficilement appréciable, donc, surtout par ceux qui en sont les auteurs !) avec le déroulé des projets dont ils sont issus. On peut, par contre, tenter d'analyser deux points : les rapports entre ce déroulé et la concrétisation de l'interdisciplinarité visée par les recherches; comment la manière dont les rencontres partenariales se concrétisent joue sur le rythme de la production de connaissances.

En ce qui concerne le premier point, la comparaison des deux projets multidisciplinaires Flexibilité et Biobacs ne manque pas d'intérêt. Pour Flexibilité, l'interdisciplinarité est constitutive du projet. La chronique rend ainsi compte des difficultés que génère cette ambition au sein de l'équipe de recherche. Ces difficultés tiennent à des problèmes pratiques de coordination des différentes opérations disciplinaires menées, mais surtout à des décalages, voire à des désaccords, sur la façon d'intégrer ces opérations à partir et autour de la notion de flexibilité. Ce qui est alors à noter, c'est le fait que, dans le traitement de ces difficultés et le règlement de ces désaccords, la confrontation avec les partenaires apparaît comme un opérateur assez déterminant de la concrétisation de l'interdisciplinarité visée. En obligeant les chercheurs à clarifier leurs points de vue respectifs et en provoquant des changements de perspective, cette confrontation va directement contribuer à l'engagement de la synthèse des diverses analyses menées. Pour ce qui est de Biobacs, l'ambition du projet est plus modeste, celui-ci se limitant à une juxtaposition d'approches disciplinaires qu'un «ingénieur assembleur » se donne pour tâche d'intégrer. Le mode d'organisation du travail de recherche, reposant sur une logique de sous-traitance et de délégation de différents volets, rend impossibles les réagencements de compétences et de problématique que les préoccupations des partenaires auraient pu pourtant induire. La dimension "frictionnelle», plus ou moins assumée (et plus ou moins assumable), des recherches en partenariat semble donc conditionner très directement la concrétisation de l'interdisciplinarité.

En ce qui concerne le rythme de la production de connaissances scientifiques, la comparaison de nos trois projets est aussi éclairante. Il est courant d'entendre dire que le partenariat ralentit le processus de recherche, qu'il le perturbe même dans la mesure où le temps de

\footnotetext{
10 On notera que cette situation a évolué depuis le moment où cet article a été rédigé, un certain nombre des publications alors soumises à des revues diverses ayant été acceptées (Compagnone, 2004 ; Lémery et al., 2005).
}

l'action n'est pas celui de la recherche. Nos observations confirment bien ce caractère heurté de l'avancement des projets, sans que l'on puisse, pour autant, attribuer ce caractère un peu chaotique à quelque chose qui serait de l'ordre du choc entre deux logiques incompatibles. $\mathrm{Si}$, dans le cas de Viticulture, le porteur du projet est confronté à un partenariat qui impose bien un certain tempo au travail, ce partenariat laisse aussi la recherche se dérouler de manière assez autonome. Dans les deux autres cas, ce sont les problèmes posés par la coordination entre chercheurs qui apparaissent prédominants. Plutôt qu'un ralentissement du processus de recherche, le partenariat apparaît surtout générer des aléas et des ruptures qui aboutissent à changer l'ordre des choses, remettent en cause des prévisions, mais contribuent aussi à assurer l'avancée même du projet, moyennant des effets imprévus, des accélérations et des inflexions tenant autant à certaines interprétations de la situation par l'une ou l'autre des parties prenantes (c'est-à-dire à certaines suppositions relatives à ce qui se passe...) qu'à des ajustements négociés, sur la base de confrontations effectives. À ce niveau, c'est la dimension « fictionnelle » des recherches en partenariat qui semble surtout opérante.

\section{La production de connaissances actionnables}

La recherche en partenariat se caractérise aussi par le souci de produire des connaissances qui soient actionnables, c'est-à-dire qui présentent une pertinence au regard des préoccupations propres des partenaires (qui ne sont pas celles des chercheurs) et qui soient susceptibles de déboucher sur des pistes d'action cohérentes avec les ressources pratiques dont ils disposent. Que peuton dire alors de la façon dont la production de telles connaissances peut être conditionnée par des effets de dispositifs?

Si les trois projets sont bien animés par une volonté d'aboutir à des connaissances utiles pour les partenaires qu'ils mobilisent, ils démarrent sans que les modalités d'appropriation des produits de la recherche aient été préalablement imaginées. Dans le projet Flexibilité, l'objectif en la matière, formulé sur un mode très général, est de contribuer à un renouvellement de la réflexion sur l'accompagnement des éleveurs. Bien que cet objectif ait été plusieurs fois réaffirmé, sa non-reprise par les partenaires conduit en fait les chercheurs à en reporter la réalisation à une phase ultérieure. Même si l'avancée propre de la recherche a bien permis de commencer à cerner quels pouvaient être les enjeux d'un conseil renouvelé, le partenariat du projet, tel que constitué, n'apparaît pas approprié pour assurer une telle traduction. Pour le projet Biobacs, la situation est différente. Celui-ci s'inscrit dès sa conception dans une perspective de recherche appliquée. Initialement, c'est sous la forme d'un simple retour 
Tableau 2. Les points-clés des dispositifs de pilotage des trois projets.

\begin{tabular}{|l|l|l|l|}
\hline & Flexibilité & Biobacs & Viticulture \\
\hline $\begin{array}{l}\text { Force propre du } \\
\text { projet }\end{array}$ & $\begin{array}{l}\text { Le collectif de recherche est } \\
\text { fortement constitué, mais la } \\
\text { question du conseil aux éle- } \\
\text { veurs reste peu mobilisatrice }\end{array}$ & $\begin{array}{l}\text { Des alliances de circonstance, } \\
\text { mais un projet qui apporte } \\
\text { des résultats rapidement ap- } \\
\text { plicables }\end{array}$ & $\begin{array}{l}\text { Un acteur régional fédérateur } \\
\text { imprime le mouvement, la re- } \\
\text { cherche s'adapte tout en gar- } \\
\text { dant une part d'autonomie }\end{array}$ \\
\hline $\begin{array}{l}\text { Individus } \\
\text { médiateurs }\end{array}$ & $\begin{array}{l}\text { La médiation s'opère grâce à } \\
\text { des acteurs périphériques (éle- } \\
\text { veurs enquêtés, technicien et } \\
\text { étudiants enquêteurs) }\end{array}$ & $\begin{array}{l}\text { Les responsables du pro- } \\
\text { jet font jouer leurs rela- } \\
\text { tions interpersonnelles dans } \\
\text { deux sphères d'action (exper- } \\
\text { tise phytosanitaire et dévelop- } \\
\text { pement agricole) }\end{array}$ & $\begin{array}{l}\text { Le } \text { (Inra) de la CRECEP assurent } \\
\text { la médiation }\end{array}$ \\
\hline $\begin{array}{l}\text { Reconfigurations } \\
\text { en cours de } \\
\text { projets }\end{array}$ & $\begin{array}{l}\text { Elles s'opèrent en interne, du } \\
\text { côté de la recherche (interdis- } \\
\text { ciplinarité) }\end{array}$ & $\begin{array}{l}\text { Un dispositif spécifique de va- } \\
\text { lorisation des résultats s'ad- } \\
\text { joint au projet }\end{array}$ & $\begin{array}{l}\text { Le projet de recherche évo- } \\
\text { lue vers un dispositif de « re- } \\
\text { cherche action » }\end{array}$ \\
\hline
\end{tabular}

d'informations vers des partenaires perçus comme utilisateurs potentiels que la question du développement est traitée dans le projet. Dans les faits, cependant, c'est l'irruption d'un partenaire inattendu, la DIREN, qui va assurer l'opérationnalisation du projet, celui-ci devenant, momentanément, le catalyseur d'une série d'échanges inédits entre les chercheurs mais aussi entre les partenaires. Dans le cas du projet Viticulture, on peut relever un processus analogue. Le tournant du projet vers l'action s'opère à un moment et suivant des modalités que ni la recherche ni les partenaires n'avaient imaginés au départ. C'est l'animateur de la CRECEP, on l'a vu, qui joue là un rôle déterminant. Sorte de "marginal-sécant », puisqu'il est en contact à la fois avec les partenaires et avec le chercheur porteur du projet, il possède une information sur la perception que les partenaires ont de l'action que n'a pas ce dernier. Relais de la façon de voir des partenaires - tout en étant aussi porteur de sa propre conception des choses -, il induit donc le mouvement, en provoquant l'organisation d'un séminaire destiné à réfléchir sur des modes d'action envisageables à partir du projet Viticulture. S'il pousse ainsi à «faire quelque chose", cet impératif semble plus lié au pas de temps qui s'est écoulé depuis le début du travail qu'à une réflexion effective sur les résultats réellement produits et sur l'usage que l'on peut en faire. Il aboutit cependant à une démarche explicite de recherche-action qui relance la dynamique proprement scientifique du projet et fournit les bases d'une articulation effective entre recherche et développement.

Au total, la possibilité pour des recherches en partenariat d'aboutir à des connaissances actionnables apparaît bien tenir à nouveau à une certaine articulation entre une dimension fictionnelle et une dimension frictionnelle. Elle suppose, d'un côté, une croyance partagée en l'idée que ce genre d'activité est bien susceptible de déboucher, à plus ou moins long terme, sur quelque chose d'effectivement utile. Et elle implique, de l'autre, que puissent advenir certains faits, certains événements, générateurs de déplacements à même de permettre les effets de connaissances recherchés du côté des partenaires.

\section{Conclusion}

L'examen de ce qui se passe effectivement entre les parties prenantes de recherches en partenariat révèle des processus d'interaction qui sont loin de relever en majorité de l'échange direct, de la discussion ouverte, de la recherche délibérée d'accords explicites sur ce que les uns et les autres ont à faire ensemble, pourquoi et comment. Ce qui ressort plutôt de notre expérience et de nos analyses, c'est l'importance de phénomènes imprévus, des rattrapages qu'ils induisent, le plus souvent à partir de l'interprétation des enjeux que les protagonistes n'ont ni le temps, ni l'occasion, ni même parfois le désir de vérifier ensemble et qui restent donc à l'état de suppositions. Tout se passe comme si, une fois affichées certaines prétentions et posés certains actes, il s'agissait surtout de faire en sorte que l'histoire continue.

Notre approche conduit ainsi à un questionnement sur le genre de gouvernance (et de compétences, aussi) que requiert le pilotage des recherches en partenariat. Sans prétendre apporter des réponses définitives à cette question, nos analyses nous permettent de dégager trois points-clés dont la maîtrise et la gestion nous semblent conditionner la conduite des recherches en partenariat (Tab. 2). Le premier est l'importance à accorder à la dynamique du projet lui-même, qui, tant du côté de la recherche que des partenaires du développement, conditionne leur capacité à entretenir la fiction tout en assumant les frictions constitutives de leur collaboration. Le second est le caractère crucial que confère la présence, dans de telles entreprises, de "médiateurs " à même, par leurs multi-appartenances, d'assurer la mise en commun des préoccupations propres à chacun des mondes. Le troisième est l'attention qu'il convient de porter à la question des reconfigurations éventuelles du partenariat 
qu'impliquent les évolutions mêmes d'un projet, les déplacements de problématique auxquels ces évolutions peuvent aboutir entraînant une modification en conséquence des collectifs pertinents pour les assumer.

En définitive, les facteurs les plus déterminants pour le déroulement de recherches en partenariat ne sont pas forcément des objectifs initiaux clairs et partagés, des plans d'action a priori détaillés ou un système de pilotage préalablement et strictement défini (toutes choses nécessaires mais non suffisantes...). Ils sont plutôt de l'ordre de ce qui est susceptible de permettre le maintien, sans dissipation, d'histoires relativement chaotiques qui sont inhérentes à ce type de recherches. Une telle conclusion est sans doute pertinente dans le domaine agricole où l'action est tributaire d'une multiplicité d'acteurs et d'interactions complexes entre nature et société. En l'état actuel de notre réflexion, nous laisserons ouvert ce questionnement.

\section{Remerciements}

Ces travaux ont bénéficié de financements de l'Inra et du conseil régional de Bourgogne dans le cadre du programme PSDR et ont été présentés au symposium «Territoires et enjeux du développement régional ", à Lyon, du 9 au 11 mars 2005 (voir http://www.inra.fr/rhonealpes/symposium/pdf/session2-1_3.pdf). Les auteurs remercient les lecteurs et les rapporteurs de cet article pour leur contribution très constructive à l'amélioration du texte.

\section{Références}

Aggeri, F., 2002. La construction des objets de recherche dans les partenariats d'exploration, in Actes du séminaire Recherches pour et sur le développement régional, Montpellier, 8-9 janvier 2002, Paris, Inra, 71-89.

Albaladejo, C., Casabianca, F. (Eds), 1997. La recherche-action. Ambitions, pratiques, projets, Études et recherche sur les systèmes agraires et le développement, 30.

Argyris, C., Schön, D.A, 1978. Organizational Learning: A Theory of Action Perspective, Addison, Wesley Publishing Company.

Bailly, A., Daucé, P., Koller, R., Lhoste, P., Manichon, H., Rosenberg, P-E., 2005. Évaluation nationale des programmes de recherche PSDR de l'INRA. Rapport de la commission d'évaluation, Inra, Paris.

Barbier, M., 1998. Pratiques de recherche et invention d'une situation de gestion d'un risque de nuisance: d'une étude de cas à une recherche-intervention. Thèse de doctorat en sciences de gestion, Université Jean Moulin, Lyon.
Béranger, C., Compagnone, C., Évrard, P., Bonnemaire, J., 2002. Recherche, agriculture, territoires : quels partenariats? Rapport, Inra, Paris.

Chia, E., Barbier, M., 1999. Gestion de la qualité de l'eau : apprentissage collectif et rôle des prescripteurs, Cahiers Agriculture, 8, 2, 109-117.

Chiffoleau, Y., Dreyfus, F., Touzard, J.-M., 2001. Chercheurs et viticulteurs partenaires pour l'innovation : interactions, institutions et apprentissages, Natures Sciences Sociétés, 9, 3, 29-36.

Compagnone, C., 2003. Les Pratiques viticoles en Bourgogne : $d y$ namiques de changement et place des pratiques respectueuses de l'environnement. Rapport d'étape du projet PSDR, Inra, Dijon.

Compagnone, C, 2004. Agriculture raisonnée et dynamique de changement en viticulture bourguignonne, in Conein, B. , Ferrand, A., Lazega, E. (Eds), Connaissance et relations sociales, Recherches sociologiques, 35, 3, 103-121.

Danguin, M., Fournier, J.-C., Hovan, M., Soulard, C.T., 2004. Efficacité, faisabilité et opportunité des biobacs : l'expérience bourguignonne. Plaquette, Inra-LISTO/MGS, Dijon.

Fournier, J.-C., Ponce, C., Thévenot, M., Dousset, S., Lemière, J.-P., Nouaïm, R., Soulard, C.T., 2004. Biobacs : évaluation et prévention des risques environnementaux. Rapport Inra-PSDR Bourgogne, Dijon.

Gadille, M., d'Iribarne, A., 2001. L'évaluation des chercheurs du CNRS : une impasse socialement construite? Communication au Forum de la régulation, Paris, 11-12 octobre.

Hatchuel A., 2000. Recherche, intervention et production de connaissances, in Actes du séminaire Recherches pour et sur le développement territorial, Montpellier, Paris, Inra, 2, 27-40.

Hervieu, B., Flamant, J.-C., de Jouvenel, H., 2003. INRA 2020. Alimentation, agriculture, environnement : une prospective pour la recherche. Rapport Inra, Paris.

Iribarne, A. d', 2005. Quelques réflexions à propos de l'exercice d'évaluation du PSDR Bourgogne, in Étude des partenariats tissés entre la recherche et le développement grâce au PSDR Bourgogne. Rapport Inra-PSDR, Dijon, 5-9.

Lémery, B., Barbier, M., Chia, E., 1997. Une recherche-action en pratique : entre production d'eau minérale et agriculture, Études et recherche sur les systèmes agraires et le développement, 30, 71-89.

Lémery, B., Bardey, H., Brossier, J., Dedieu, B., Dégrange, B., Ingrand, S., 2003. Évolutions de la filière bovine et "flexibilité» des exploitations d'élevage : études de cas dans le bassin allaitant bourguignon. Rapport Inra, Dijon.

Lémery, B., Ingrand, S., Dégrange, B., Dedieu, B., 2005. Agir en situation d'incertitude : le cas des éleveurs de bovins allaitants, Économie rurale, 288, 57-69.

Sebillotte, M., 1996. Les Mondes de l'agriculture : une recherche pour demain, Paris, INRA Éditions.

Sebillotte, M., 2000. Des recherches pour et sur le développement local : partenariat et transdisciplinarité, Revue d'économie régionale et urbaine, 3, 535-556.

Segrestin, B., 2003. La Gestion des partenariats d'exploration. Thèse de l'École nationale supérieure des mines de Paris. 